This item was submitted to Loughborough's Research Repository by the author.

Items in Figshare are protected by copyright, with all rights reserved, unless otherwise indicated.

\title{
Solvent-free solid-state-processed tapes of ultrahigh-molecular-weight polyethylene: influence of molar mass and molar mass distribution on the tensile properties
}

\section{PLEASE CITE THE PUBLISHED VERSION}

http://dx.doi.org/10.1021/acs.iecr.5b01469

\section{PUBLISHER}

(c) American Chemical Society

\section{VERSION}

AM (Accepted Manuscript)

\section{PUBLISHER STATEMENT}

This work is made available according to the conditions of the Creative Commons Attribution-NonCommercialNoDerivatives 4.0 International (CC BY-NC-ND 4.0) licence. Full details of this licence are available at: https://creativecommons.org/licenses/by-nc-nd/4.0/

\section{LICENCE}

CC BY-NC-ND 4.0

\section{REPOSITORY RECORD}

Ronca, Sara, Giuseppe Forte, Hendrik J. Tjaden, and Sanjay Rastogi. 2016. "Solvent-free Solid-stateprocessed Tapes of Ultrahigh-molecular-weight Polyethylene: Influence of Molar Mass and Molar Mass Distribution on the Tensile Properties". figshare. https://hdl.handle.net/2134/21924. 
Solvent-free, solid state processed tapes of UHMWPE: influence of molar mass and molar mass distribution on tensile properties

Sara Ronca ${ }^{a}$, Giuseppe Forte ${ }^{a}$, Hendrik Tjaden ${ }^{b}$, Sanjay Rastogi, ${ }^{a, b^{*}}$

a) Department of Materials, Loughborough University, Leicestershire LE11 3TU, England (UK).

b) Research Institute, Teijin Aramid B.V., Velperweg 76, Arnhem, The Netherlands.

*Corresponding author: Sanjay Rastogi: s.rastogi@ lboro.ac.uk.

In normal practice, ease in processing comes at the expense of reduction in mechanical properties. Here we show that by controlled synthesis, it is possible to synthesize a wide range of linear Ultra High Molecular Weight polyethylenes that can be stretched uniaxially into tapes. In these uniaxially drawn tapes the bundles of fibers align themselves in a preferred crystal plane orientation accounting for an extraordinary high tensile modulus. The stretching process is accomplished in the solid state, with no need for any solvent. The ease in solid state processing provides a unique opportunity to follow the influence of molar mass on tensile properties. The uniaxially drawn tapes, similar to the fibers spun from solvent, confirm the empirical relationship between tensile strength and tensile modulus proposed by van Krevelen in 1976 and subsequently supported by experimental findings on solution spun fibers by Smith and Lemstra. However, the solid state processed tapes do not achieve the high values of tensile strength expected from this relationship, where a modulus of 200GPa would correspond to a tensile strength of $5 \mathrm{GPa}$. The relatively lower tensile strength of $4 \mathrm{GPa}$ for the extraordinary high modulus of $200 \mathrm{GPa}$ observed in the uniaxially stretched tapes is attributed to the presence of defects, as tapes can be considered composite of fibers. 


\section{The high modulus in combination with the tensile strength in tapes has the potential to provide unique physical properties in composites.}

Semi-crystalline polymers can be considered as a matrix of heterogeneously distributed entanglements, where the disentangled regions reside in the crystalline domains and the entangled regions in the amorphous domains. The presence of entanglements is realized during solid state deformation of the semi-crystalline polymer, where the strain hardening slope in the stress-strain curve is considered to be representative of the entangled state in the amorphous region. It is well accepted that crystallization conditions of semi-crystalline polymers have a strong influence on entanglements formation in the amorphous region. For example, in Ultrahigh molecular weight polyethylene having molar mass greater than 1 million $\mathrm{g} / \mathrm{mol}$, on crystallization from dilute solutions, the number of entanglements can be reduced to such an extent that the deformation proceeds with neck formation showing nearly no strain hardening, and the material could be drawn more than 300 times of its initial length. When the same material is melt crystallized, the presence of entanglements in the amorphous regions leads to a strong strain hardening slope, causing the breakage of the material after a draw ratio of approximately 10 times only [1]. The dramatic differences in the deformation process and its correlation with the crystallization conditions are attributed to the nature of entanglements formation, having implications on chain folding, that can happen according to the adjacent reentry or to the switch board model [2]. These differences have been addressed by solid state NMR [3].

The influence of entanglements in the solid state deformation of UHMWPE has been investigated by several authors in the past. In a series of publications it has been conclusively shown that, by reducing the chain overlap concentration on dissolution of the polymer in a suitable solvent, followed by crystallization on solvent evaporation, the draw ratio in solid state of the semi-crystalline polymer can be increased substantially. The studies lead to the 
establishment of an empirical relationship between the maximum achievable draw-ratio $\left(\lambda_{\max }\right)$ and the polymer concentration in a theta solvent (Equation 1) [4, 5],

(1) $\lambda_{\max } \propto\left(c M_{w}\right)^{-0.5}$

The achieved draw ratio has implications on the tensile properties, tensile strength and tensile modulus of the polymer, according to Equations 2 and 3 [4]

(2) $\sigma=A-\frac{B}{\overline{M_{n}}}$

(3) $\sigma=m E^{n}$.

Smith, Lemstra and co-workers further demonstrated that on crystallization just above the critical overlap concentration $c^{*}$, tensile properties increases with the increasing molar mass, while below this concentration brittle, incoherent films are produced. The experiments performed on solution spun fibres were extended to understand the fracture mechanism that unraveled limitations in achieving the ultimate properties, in terms of tensile strength and modulus. However, since the chosen polymers were synthesized using heterogeneous Z-N catalysts the number average molar masses, $M_{n}$, of the investigated polyethylenes were between $50^{*} 10^{3} \mathrm{~g} / \mathrm{mol}$ to $500^{*} 10^{3} \mathrm{~g} / \mathrm{mol}$ and the weight average molar masses, $M_{\mathrm{w}}$, varied between 0.12 to $4.5^{*} 10^{6} \mathrm{~g} / \mathrm{mol}$. The presence of low molar mass component often led to an increased number of chain ends and a reduction in the apparent crystal length without defects in the drawn fibers. Moreover, the presence of chain ends also facilitated the creep of the drawn material, leading to a decrease in the tensile strength to break compared to the theoretical value. One of the limiting factors in obtaining the disentangled state via the dissolution route has been the polymer molar mass. For example, to achieve the same draw ratio with an increase in molar mass by a factor of two, the polymer concentration in the solvent has to be reduced by the same factor. Dissolution will become more challenging when the polydispersity is decreased further. An alternative way to overcome this limiting factor is to control the entanglements during the 
polymer synthesis itself. Such possibility was recognized by Smith et al. earlier [6] but could not be brought to a level of practical significance due to limitations in polymer synthesis, especially because of the low polymerization temperatures and catalyst activity. Recently, it has been shown by some of us [7] that by making use of a single-site homogeneous catalytic system, it is possible to control the polymerization rate against the crystallization rate, in order to achieve a powder having lower bulk density that can be directly compressed below its meting point into homogeneous films, with no need to dissolve it in a solvent. These films can be drawn by more than 300 times [8] without melting the polymer, in a broad temperature window below the equilibrium melting temperature of the polymer. Rheological studies [9] also show that the entanglement density decreases with increasing molar mass, where by increasing the polymerization time, the molar mass $M_{\mathrm{w}}$ can be varied from 0.5 to 9.0 million $\mathrm{g} / \mathrm{mol}$. The singlesite catalytic system also provides a narrow molecular weight distribution (MWD). After polymerization, since no solvent is required for the solid state deformation, these materials provide a unique opportunity to overcome the stringent requirement of reduction in polymer concentration with increasing molar mass, (see equation 1), and an opportunity to perform a systematic study on the influence of weight and number average molar mass on the tensile properties.

Though it is of academic and technological interest to compare the fibres obtained by solution route and the ensemble of fibres in the form of tape obtained by our solvent-free route, caution during comparison must be taken. One should bear in mind that the tape we obtain is a composite of elongated fibres, thus appropriate comparison could only be realized by analyzing fibres from the tape. In this publication, we have not made any such attempt and have made use of the immense knowhow available on solution spun fibers [10] (or solution route for making fibres) in the understanding of tapes obtained via the solvent-free route. The comparison between fibres obtained from solvent-free route and solution-spun ones will be the subject of a separate study and is beyond the scope of this publication. 


\section{Results and Discussion}

For the present studies a set of samples have been synthesized using the same catalytic system and the same general reaction conditions reported in reference 8 and in the experimental section. The solvent of choice when using single-site homogenous catalyst activated by MAO is usually toluene, because of the ease in dissolution of the catalytic system and its relatively high dielectric constant. A higher dielectric constant can lead to the formation of solvent-separated catalyst and co-catalyst ion pair, instead of contact ion-pair, thus ensuring a higher catalytic activity when compared to solvents with a lower dielectric constant, such as heptane [11]. For these reasons we have focused our attention on samples of UHMWPE synthesized in toluene; however, additional samples synthesized from heptane and from a mixture of toluene/heptane are reported later on in the text for comparison.

Table 1 summarizes a set of samples synthesized using the same polymerization conditions (reaction medium: toluene; temperature: $10^{\circ} \mathrm{C} ; 1$ bar monomer pressure) while varying the polymerization time. Independently of the polymerization time, these polymers are easy to compress below the melting temperature, achieving homogenous film that do not show grain boundaries between the original polymer particles. These compressed films can be stretched uniaxially in a broad temperature window ranging from 120 to $140^{\circ} \mathrm{C}$. It should be noted that commercial samples of UHMWPE can be uniaxially stretched in the solid state, but this procedure requires a very careful control of the temperature in a range of just $1-2{ }^{\circ} \mathrm{C}$ below the melting temperature of the polymer The sample name is in the form PE_xM_y, where $x$ represent the $M_{\mathrm{w}}$ and y the MWD as obtained from rheological characterizations. 
Table 1. Molecular characteristics of the UHMWPE samples synthesised in toluene at various times $\left(\mathrm{t}_{\mathrm{p}}\right)$ The samples PE_2M_2.1 and PE_2M_2.4 are similar in molar mass and molar mass distribution. However, the differences between the molar masses have implications on the mechanical properties that become evident later in the manuscript, for example figure 5.

\begin{tabular}{|l|l|l|l|l|}
\hline Sample name & $\mathbf{t}_{\mathbf{p}}(\mathbf{m i n})$ & $\boldsymbol{M}_{\mathrm{w}}\left(\mathbf{1 0 ^ { 6 }} \mathbf{g} / \mathbf{m o l}\right)$ & $\boldsymbol{M}_{\mathbf{n}}\left(\mathbf{1 0 ^ { 6 }} \mathbf{g} / \mathbf{m o l}\right)$ & $\mathbf{M W D}$ \\
\hline PE_1M_1.8 & 5 & 0.97 & 0.54 & 1.8 \\
\hline PE_2M_2.1 & 10 & 2.16 & 1.02 & 2.1 \\
\hline PE_2M_2.4 & 15 & 2.37 & 0.96 & 2.4 \\
\hline PE_4M_2.5 & 30 & 4.19 & 1.64 & 2.5 \\
\hline PE_6M_3.2 & 60 & 5.63 & 1.73 & 3.2 \\
\hline PE_7M_3.0 & 120 & $\sim 7.11$ & $\sim 2.32$ & $\sim 3.0$ \\
\hline
\end{tabular}

Molecular characteristics of the synthesized polymers:

Following the methods described elsewhere [9], with the help of a strain-controlled rheometer Ares G2 at a fixed strain in the visco-elastic region, the increase in modulus of the polymer melt under isothermal conditions is followed. The increase in modulus is representative of the increasing number of entanglements in the melt as it gradually transforms from a metastable disentangled to a thermodynamic stable, fully entangled state. Figure 1 shows the build-up in modulus as a function of time. The differences in the initial modulus recorded just after melting, $\mathrm{G}_{\mathrm{t}=0}^{\prime}$, for different polymerization times are representative of the different density of entanglement obtained during polymerization. Higher initial modulus $\left(G_{t=0}^{\prime}\right)$ for the short polymerization time means higher entanglement density created during the initial stages of polymerization. As the polymerization time increases, the entanglement density decreases. 
These observations are in accordance with our earlier publication [9], where we attributed the decrease in entanglement density with the increasing polymerization time to the suppression in nucleation barrier for crystallization of the growing chain. Thus the considerably suppressed crystallization time hinders the entanglement formation between the growing chains, and dilutes the density of entanglements formed at the initial stages of polymerization. The increase in modulus build up time with increasing polymerization time is also indicative of the increasing molar mass.

For molar mass determination, frequency sweep experiments have been performed in the melt state of the polymer, after reaching its thermodynamic equilibrium state [12].

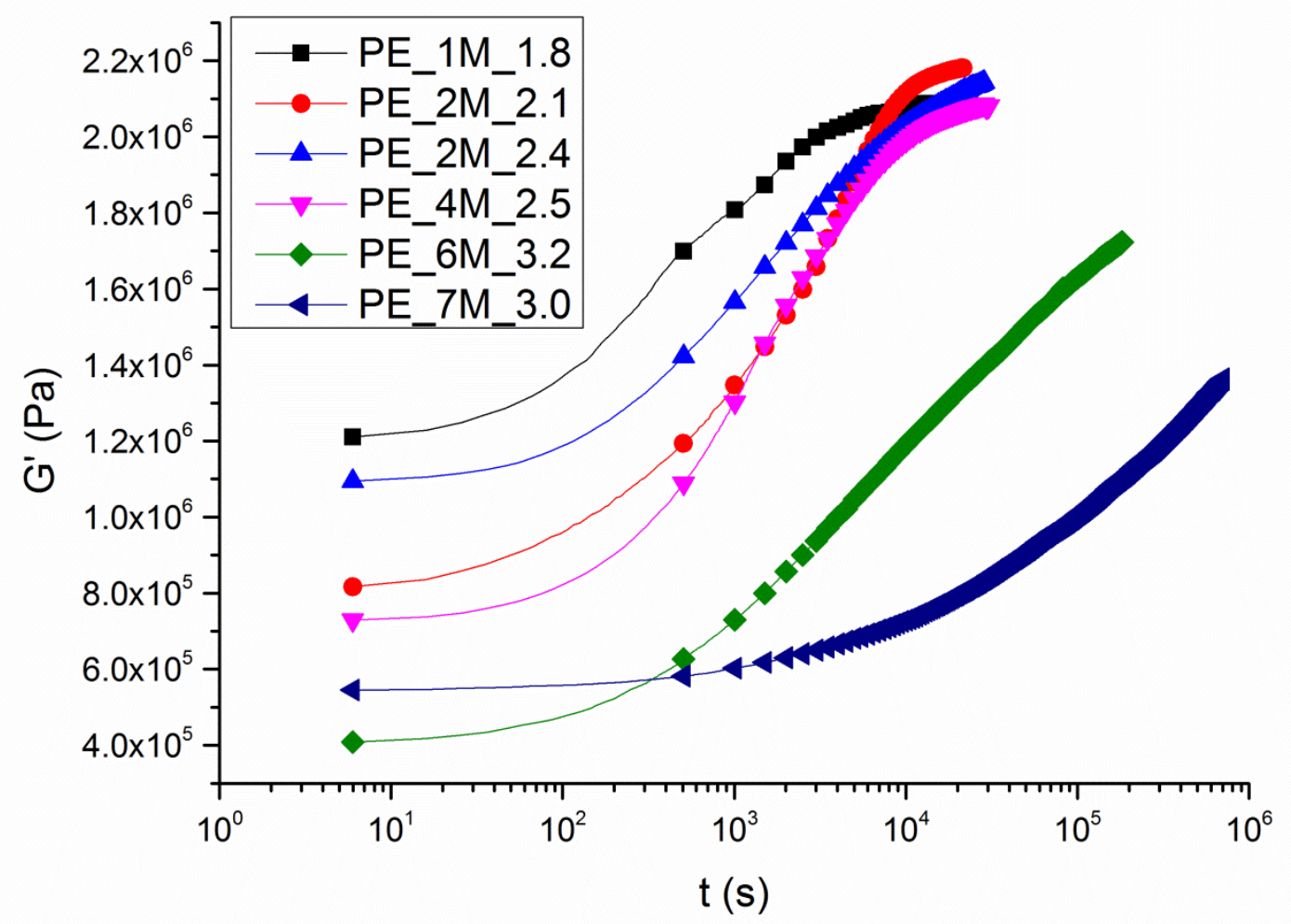

Figure 1. Modulus build-up as a function of time for samples synthesized at different reaction times.

Frequency sweep data summarized in Figure 2 shows that the cross-over point between the loss and the storage modulus shifts to lower frequencies with increasing polymerization time. 
From the frequency sweep curves, molar mass and molar mass distribution are estimated using Meads model, using built-in TA instrument software. The estimated molar mass and molar mass distributions are reported in Table 1 and Figure 3.

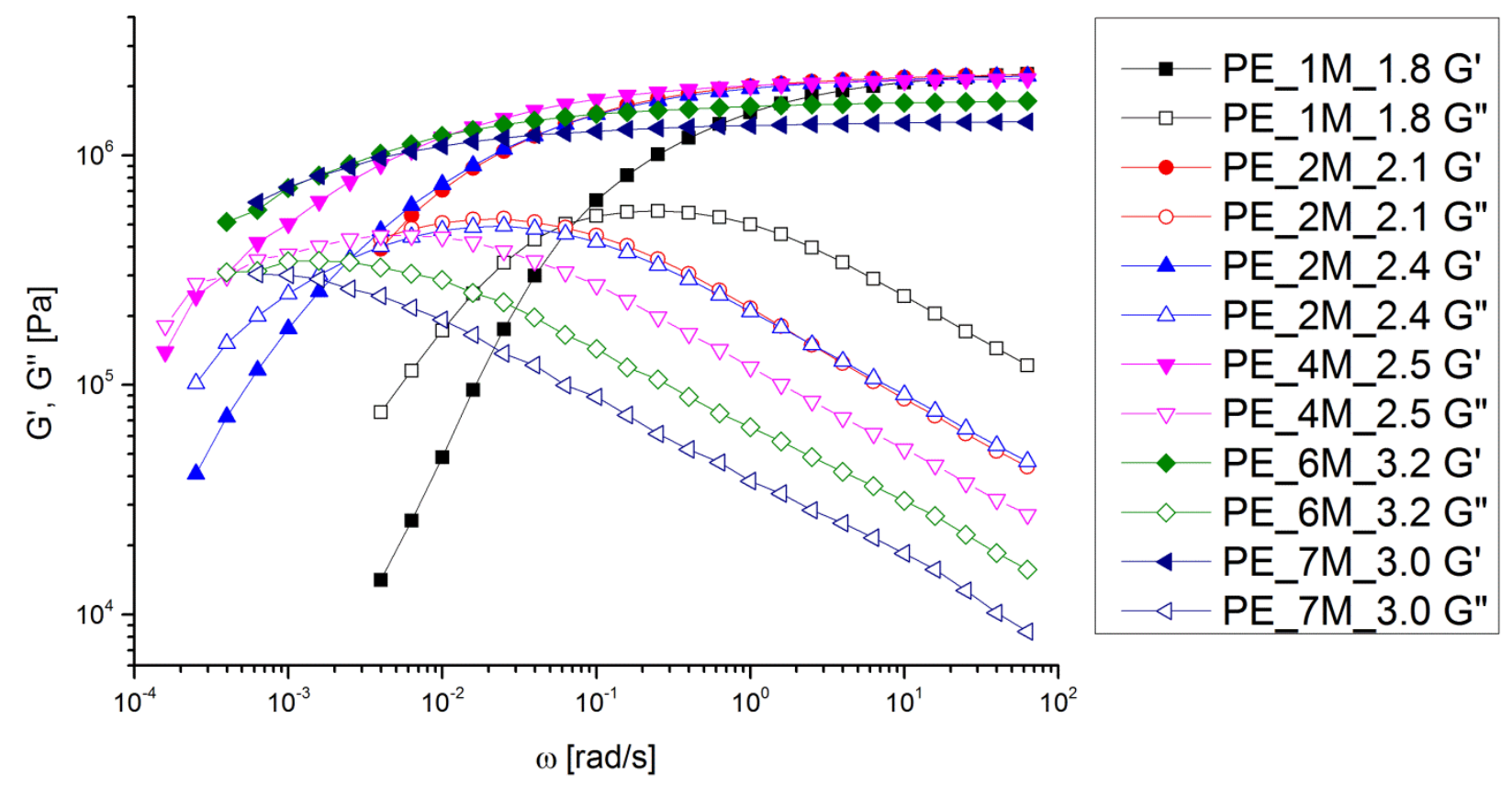

Figure 2. Frequency sweep data showing the progressive shift of the cross-over point to lower frequencies with increasing $M_{\mathrm{w}}$ 


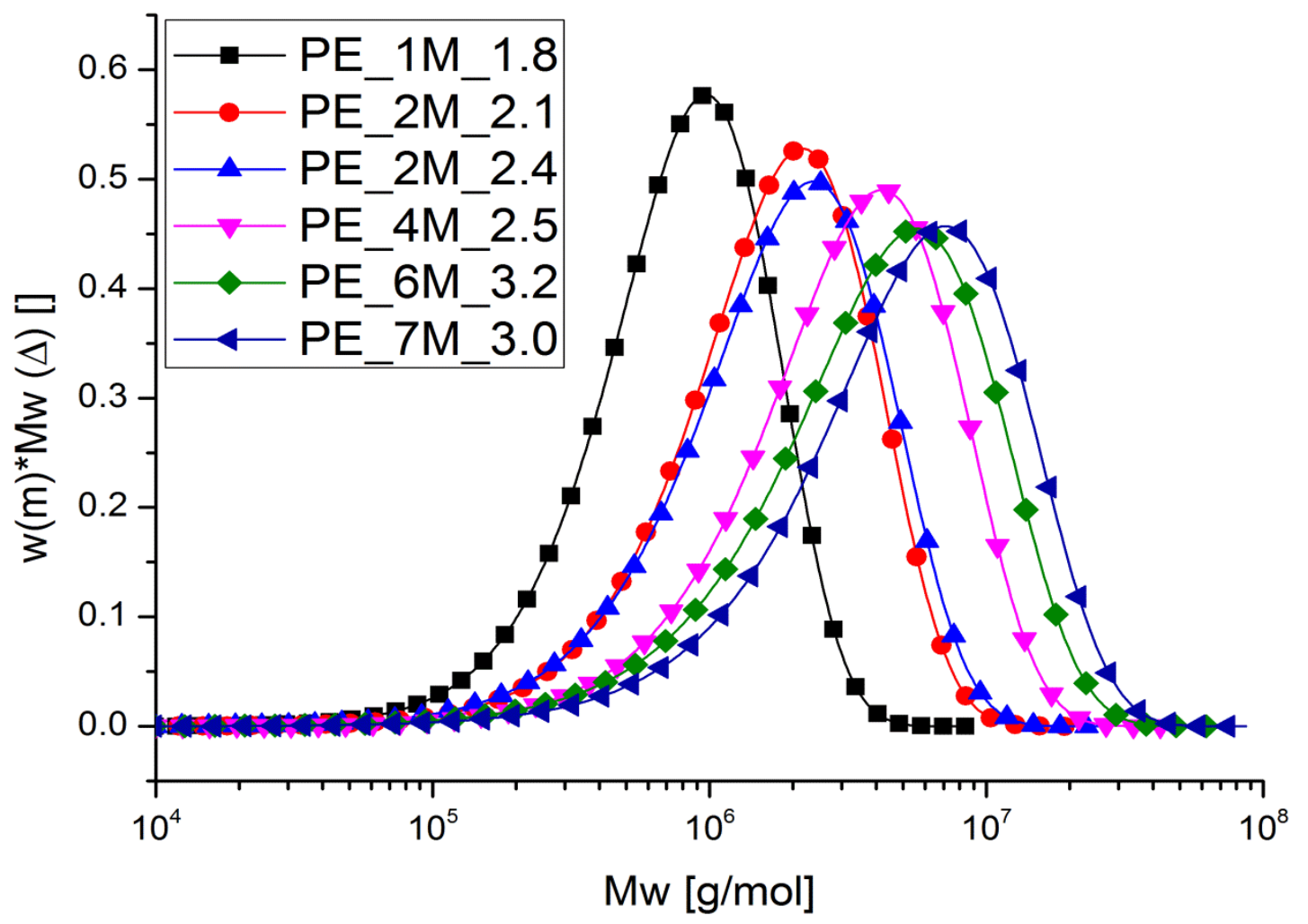

Figure 3. Molar mass distribution curves for the polymer of Table 1, as obtained from frequency sweep data.

The shift of the cross-over point to the lower frequencies is a clear indication of the increasing tube renewal time, where the tube renewal time in the polymers having molar mass greater than $200.000 \mathrm{~g} / \mathrm{mol}$ scales with $M_{\mathrm{w}}{ }^{3}$ (Figure $4 \mathrm{a}$ ) and the modulus build-up time scales with $M_{\mathrm{w}}{ }^{2.6}$ (Figure 4b), as shown in previous publications.[9, 13] 

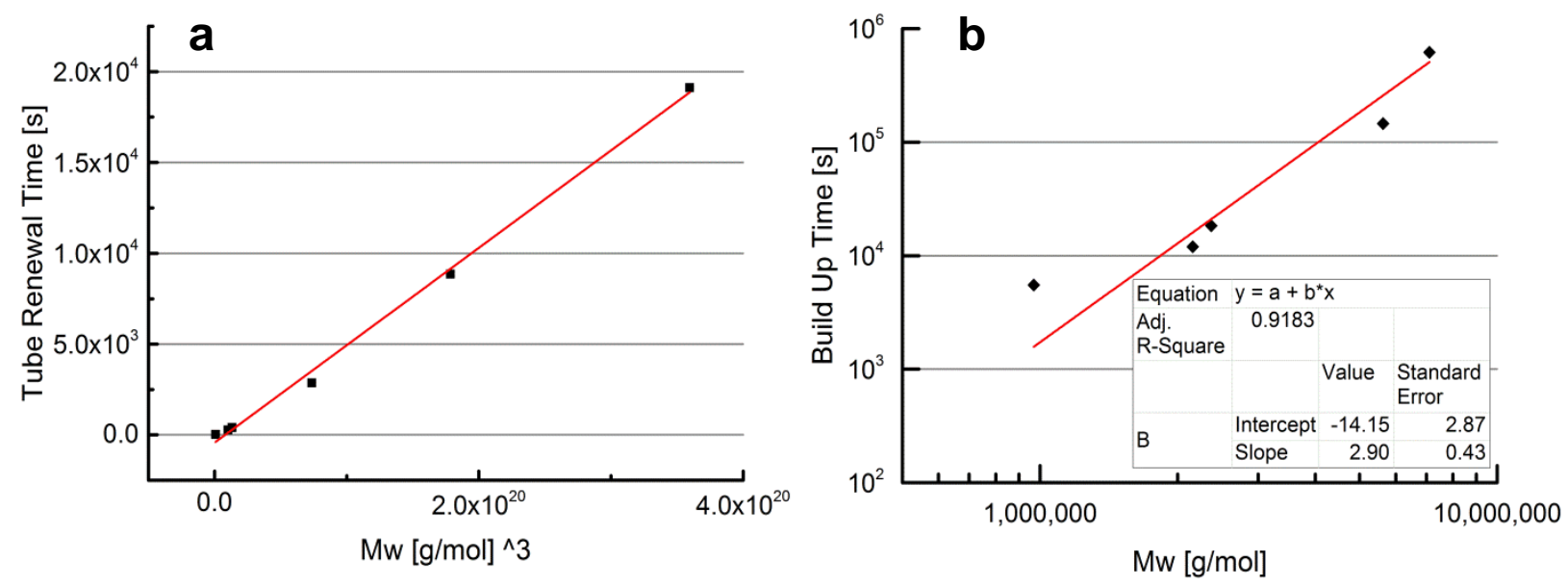

Figure 4 a) tube renewal time (obtained from the frequency cross-over point) as a function of $M_{w}{ }^{3}$ and b) modulus build up time (time by which the polymer modulus has reached the plateau region) as a function of $M_{w}$.

\section{Influence of molar mass on tensile strength and modulus:}

The failure mechanism of the uniaxially drawn tapes or fibres on application of force is a subject that needs well characterized samples for a systematic study. In the past, such experimental studies required the use of solvent to control the entanglement density in the amorphous region, where the relationship between molar mass and polymer concentration was of prime importance to unravel the influence of molar mass on mechanical properties, (as seen from equation 1) . The presence of a broad molar mass distribution, i.e. of a low number average molar mass $\left(M_{n}\right.$ $<0.5$ million $\mathrm{g} / \mathrm{mol}$ ), inherent to the polymers synthesized using a heterogeneous $\mathrm{Z}-\mathrm{N}$ catalytic system, was unavoidable. In spite of these limitations, considerable insight on the failure mechanism of fibres has been gained. There is a general consensus that the failure mechanism involved on the application of load strongly depends on experimental temperature, strain rate and molecular weight of the drawn polymer. For example, with decreasing temperature and increasing strain rate, the tensile strength increases. These observations lead to differentiation in the failure mechanisms, where two main mechanisms were proposed:1) breakage between the $\mathrm{C}-\mathrm{C}$ bonds or 2) slippage between the planes i.e. creep. Making use of theoretical models, 
Smith et al concluded that with increasing molar mass, the failure mechanism involved in breakage of fibres becomes lesser dependent on the strain rate and creep [14, 15].

The use of a single-site catalytic system provides the unique possibility of synthesizing disentangled UHMWPE samples having $M_{\mathrm{n}}$ greater than a million $\mathrm{g} / \mathrm{mol}$, that can be drawn in the solid state (below the equilibrium melting temperature) without using any solvent. From Table 1 it is apparent that $M_{\mathrm{w}}$ as well as $M_{\mathrm{n}}$ increase with the increasing polymerization time. These polymers have the potential to give us further insight on the influence of $M_{\mathrm{w}}$ and $M_{\mathrm{n}}$ on tensile strength and modulus of the uniaxially drawn tapes. What follows are the observations on tensile strength and modulus with increasing molar mass and their correlation with the molecular structure.

Figure 5 shows the effect of increasing the draw ratio on the tensile strength of the disentangled UHMWPE tapes. It is apparent that the draw ratio has a profound effect on the tensile strength, where the strength increases steeply at the initial draw ratios and enters the plateau region above the critical draw ratio. Considering that the drawing process results in crystal orientation followed by chain extension, as previously shown by us in reference 8 , the steep increase in tensile strength is attributed to chains that rapidly attain the extended chain conformation, and once most of the chains are in the extended chain conformation, further drawing has little effect, so that the plateau region is observed. The critical draw ratio beyond which tensile strength enters into a plateau region seems to vary with the increasing molecular weight. For PE_2M_2.1 (10 minutes polymerization time) the critical draw ratio is $~ 100$ while for the highest polymerization time (i.e. 120 min.), PE_7M_3.0, the critical draw ratio is $\sim 170$. The increase in the critical draw ratio with polymerization time is attributed to increase in molar mass, i.e. the longer the chains, the more it can be drawn before it reaches the critical value where no further deformation could be realized. 


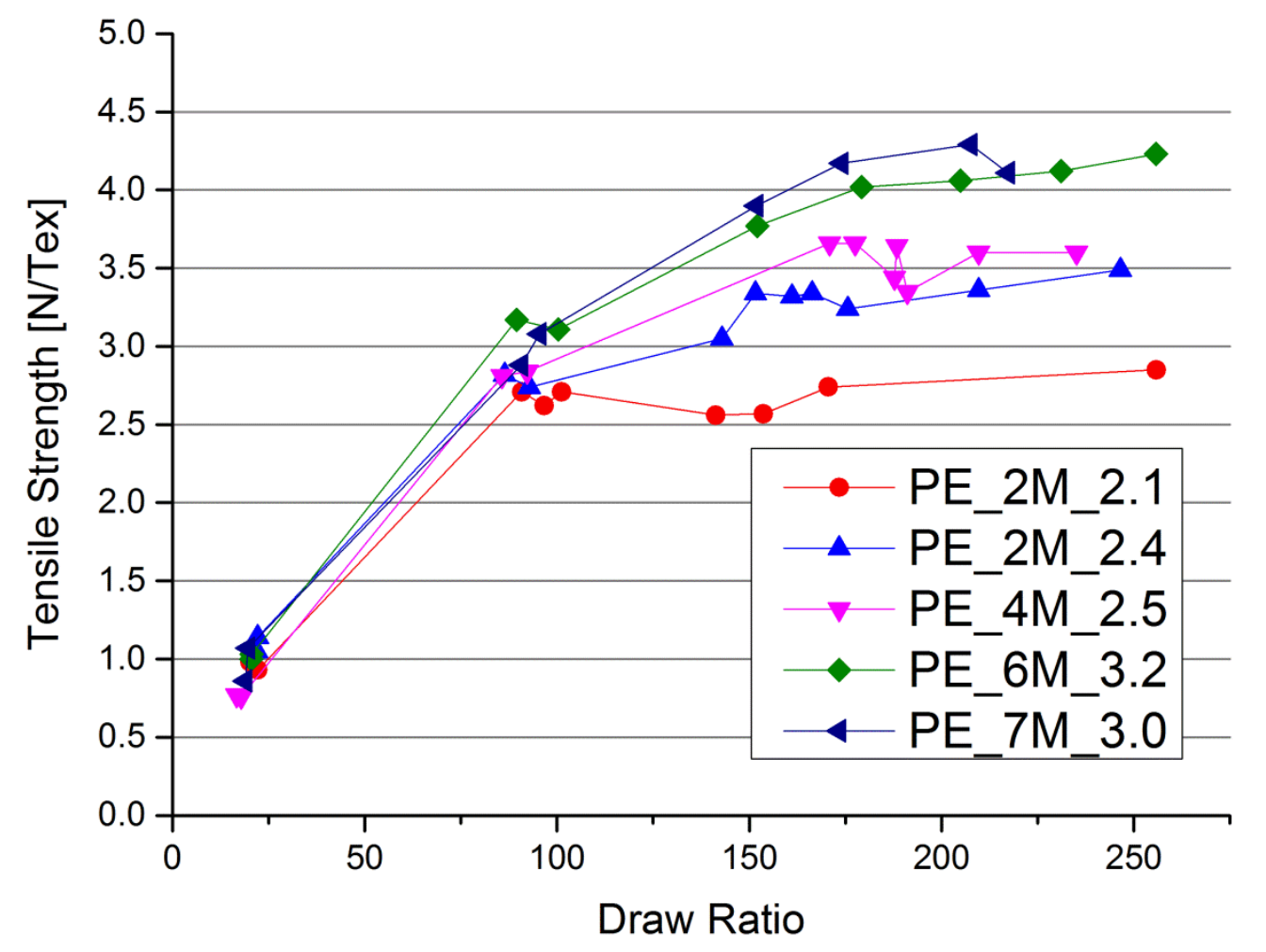

Figure 5. Influence of draw ratio on tensile strength of disentangled UHMWPE tapes. (Tex is defined as the mass in grams $\backslash 1000$ meters). Lines are drawn for visual guidance only.

The fact that at the low draw ratios $(\sim 20)$, the chain length seems to have no effect on tensile strength can be related to the crystal orientation [8]. On progressive deformation, the amorphous region - having lower modulus than the crystalline region - is likely to be stretched first, followed by pulling of chains from crystals having chain folding. Thus, longer the chain length, more it could be drawn before a plateau could be achieved.

Figure 6 shows the increase in tensile modulus with increasing draw ratio. The tensile modulus increases steeply at the initial stages before reaching a plateau value. Unlike the tensile strength, the critical draw ratio for entering the plateau region seems to be independent of the polymerization time (or molecular weight). These findings are in accordance with the earlier studies on solution-spun fibres obtained after dissolution of the polymer, reported by Savitsky et 
al. [16]. These authors also showed that the tensile modulus reaches a plateau above the draw ratio of 100 .

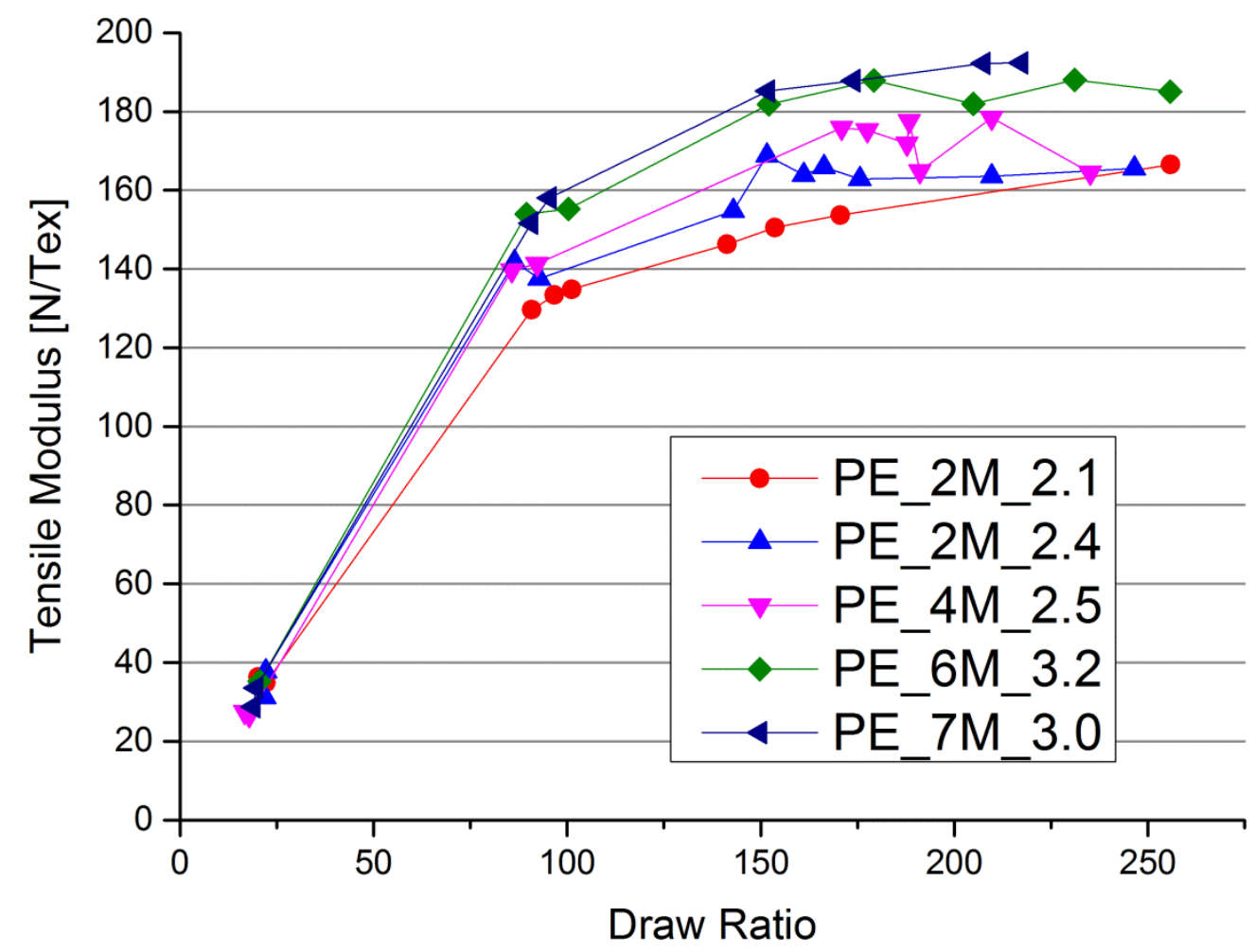

Figure 6. Influence of draw ratio on tensile modulus (N/Tex) for different polymerization time (Tex is defined as the mass in grams per 1000 meters). Lines are drawn for visual guidance only.

From figure 6 it is also evident that at the low draw ratio of $\sim 20$ there seems to be no influence of molecular weight on the tensile modulus, while at higher draw ratios the tensile modulus does increase with increasing molecular weight, which is in contradiction with the results on fibres obtained from the solvent route [17]. It should be noted that similar draw ratios do not imply that the same level of chain orientation and extension have been achieved in different samples. Major effects of drawing are in terms of crystallite size and orientation of the extended chain crystals, which are likely to vary with the increasing molar mass. A major difference between the polymers used earlier (synthesised by Z-N catalyst) and ours (synthesised using a single-site 
catalytic system) is the number average molar mass $M_{n}$, higher in our case, and thus associated with a lower polydispersity for these UHMWPEs.

To have further insight on the influence of molecular weight and molecular weight distribution, we performed the synthesis of additional samples of disentangled UHMWPE using different reaction solvents, such as heptane and a mixture of heptane/toluene. Heptane is usually related to a lower polymerisation activity in the case of homogeneous catalytic systems, that has been attributed to the lower dielectric constant of this solvent. We chose this solvent to reduce the catalytic activity in order to produce polymers of lower $M_{w}$ and $M_{n}$ in the same polymerisation times used for toluene. The polymerisation experiments in a mixture of heptane and toluene were performed in order to achieve an intermediate situation but, to our surprise, the catalytic activity in the mixture was found to be higher than in pure toluene.

In Table 2 are reported the values of $M_{\mathrm{n}}, M_{\mathrm{w}}$ and MWD obtained for samples synthesised at different times in various solvents.

Table 2. Molecular characteristics of the UHMWPE samples synthesised in heptane $(H)$ and heptane/toluene (25/75 volume ratio) mixture (Mix).

\begin{tabular}{|l|l|l|l|l|l|}
\hline Sample name & $\mathbf{t}_{\mathrm{p}}$ (min.) & Solvent & $\boldsymbol{M}_{\mathrm{w}}\left(\mathbf{1 0}^{\mathbf{6}} \mathbf{g} \mathbf{\text { mol }}\right)$ & $\boldsymbol{M}_{\mathrm{n}}\left(\mathbf{1 0 ^ { 6 }} \mathbf{g} \mathbf{\text { mol }}\right)$ & $\mathbf{M W D}$ \\
\hline PE_2M_2.9 & 30 & $\mathrm{H}$ & 2.0 & 0.7 & 2.9 \\
\hline PE_4M_2.8 & 30 & Mix & 3.9 & 1.4 & 2.8 \\
\hline PE_3M_3.0 & 60 & $\mathrm{H}$ & 3.1 & 1.0 & 3.0 \\
\hline PE_7M_3.8 & 60 & Mix & 6.9 & 1.8 & 3.8 \\
\hline PE_4M_3.4 & 120 & $\mathrm{H}$ & 4.1 & 1.2 & 3.4 \\
\hline PE_8M_3.4 & 120 & Mix & 7.5 & 2.2 & 3.4 \\
\hline
\end{tabular}


Figure 7 compares the molar mass and molar mass distribution of the polymers synthesised using the three solvent media. Considering that the same amount of catalyst and co-catalyst was used and the polymerisation temperature and pressure were kept constant $\left(10^{\circ} \mathrm{C}\right.$ and 1 bar), the increase in molar mass while changing the polymerisation medium is representative of variation in catalyst efficiency, that is currenlty being investigated. We have tentatively ascribed the higher activity observed in the solvent mixture to the fact that the presence of heptane could possibly favour a faster ethylene uptake (resulting in a higher instantaneous concentration of monomer) compared to pure toluene, but studies are in progress to verify this hypotesis and they will be the subject of a following publication. However, to have more insight on the influence of molar mass and molar mass distribution on tensile properties these polymers have been considered in this study. The different behaviour of the catalytic system in the different polymerisation media provided us with the unique opportunity to synthesise a range of polymers with varying number and weight average molar masses. It is important to notice that for all samples, inspite of differences in polymerisation medium, we observed the expected modulus build up during rheological measurements, that confirms the disentangled nature of the polymers obtained during controlled synthesis. Similar to the samples from toluene, all these samples can be drawn in the solid state to obtain tapes having high modulus and high tensile strength. 


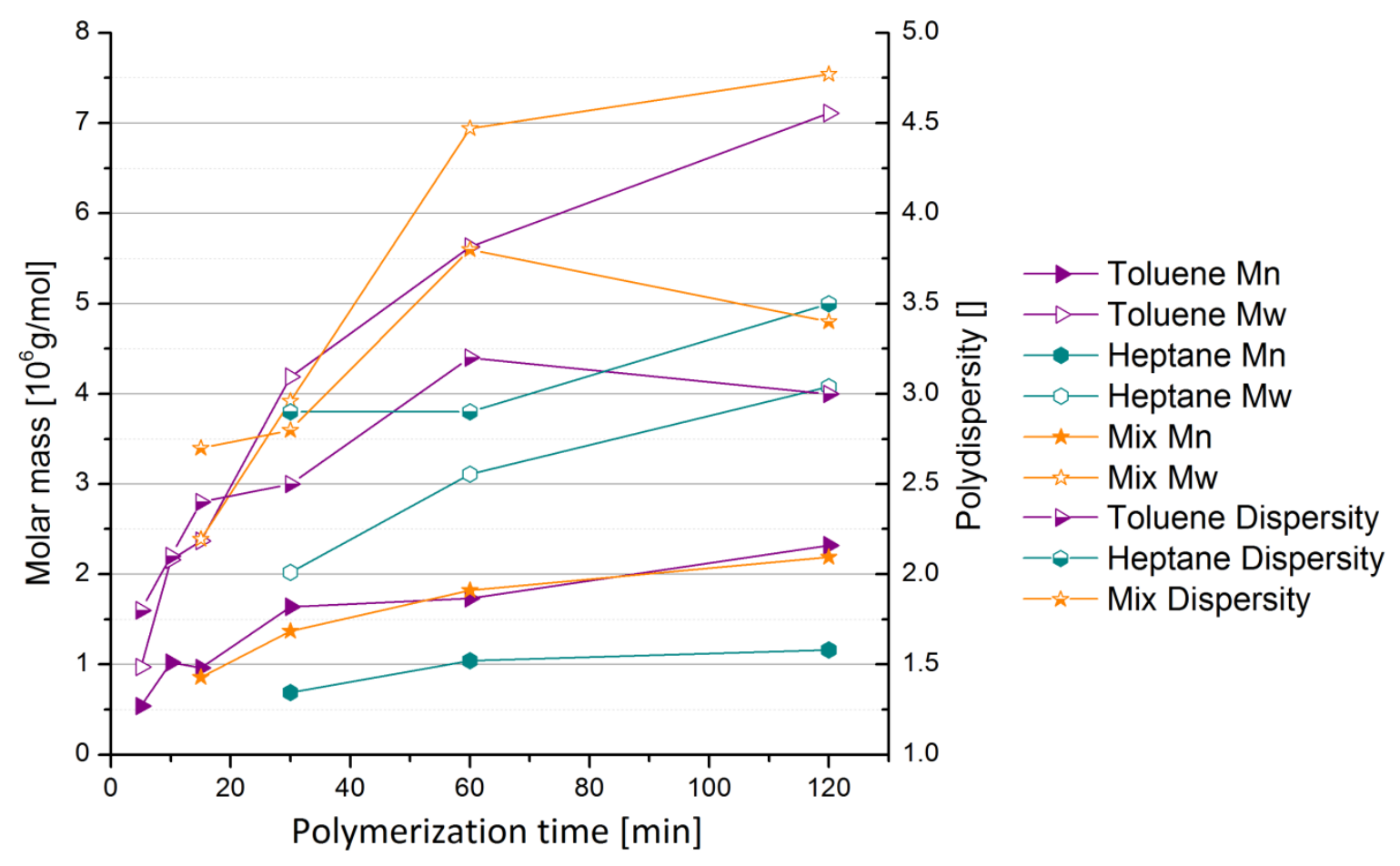

Figure 7. Overview of molar masses and molecular weight distribution for all the samples considered in this study.

Figure 8 compares the tensile strength (breaking tenacitiy) and tensile modulus of the samples polymerised using different polymerisation media.

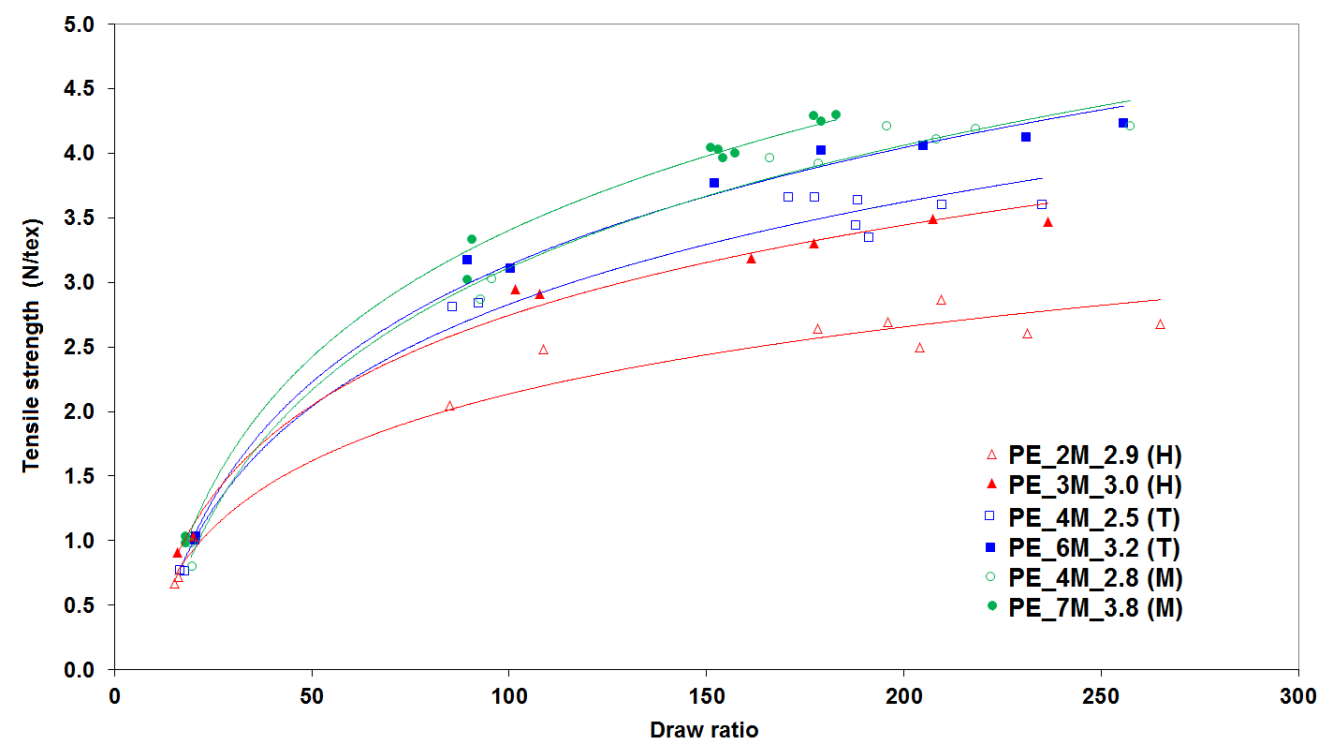




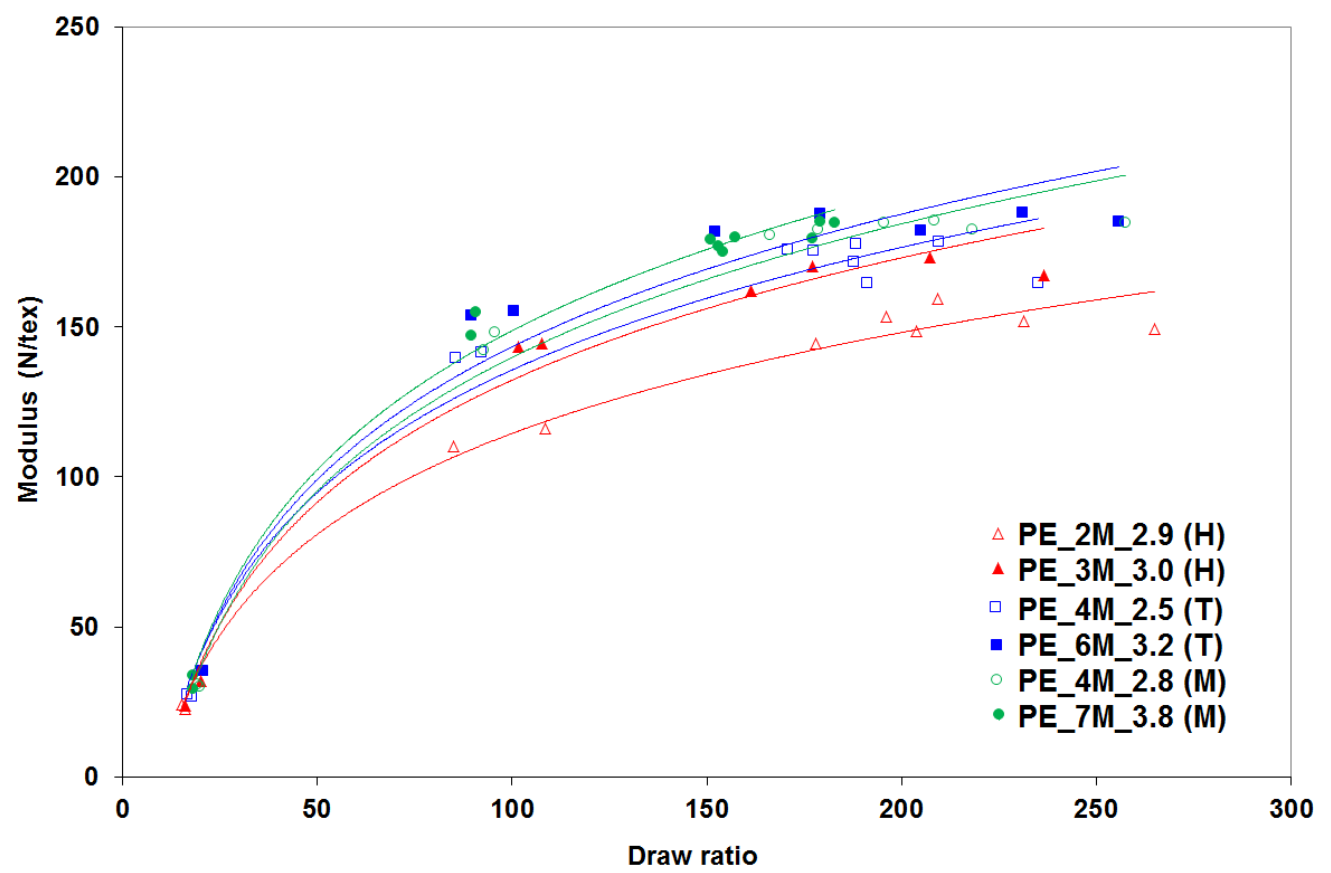

Figure 8. Tensile strength (top) and modulus (bottom) as a function of draw ratio for samples obtained from $(\mathrm{H})$ heptane, $(\mathrm{T})$ toluene and $(\mathrm{M})$ mixture of both solvents. For clarity, only the samples from 30 and 60 minutes reaction are shown.

The figure clearly demonstrates that the polymers obtained from heptane, having a lower molecular weight, show poorer tensile strength and modulus compared to the polymers obtained from the mixture. In general, the tensile strength shows stronger dependence on the molar mass, independent of the polymerization condition, while for the modulus it appears that above a certain value of $\mathrm{Mw}(\mathrm{Mw} \geq 4$ million $\mathrm{g} / \mathrm{mol})$ there is no significant enhancement of properties with increasing the molecular weight. This generalization has limitations when the differences in molar mass are not significant as is the case for the two samples PE_4M_2.5 and PE_4M_2.8 synthesised in two different solvents. Since both tensile strength and tensile modulus are characteristics of crystal, influenced by molar mass and molar mass distribution, it will be of relevance to have insight on the relationship between tensile strength and tensile modulus for different molecular weights. To recall, in fibres made via solution route Smith and Lemstra showed a linear relationship 
(Equation 3) between tensile strength and tensile modulus, where $E$ is the Young's modulus, $m$ and $\mathrm{n}$ are the constants specific to the molecular characteristics of the polymer used. Following this relationship, the authors concluded that a polymer having a molar mass as low as $M_{\mathrm{w}}=1.5$ $\mathrm{x} 10^{6}$ and $M_{\mathrm{n}}=2 \times 10^{5} \mathrm{~g} / \mathrm{mol}$ has the potential to make fibres having tensile strength above 5 GPa for the tensile modulus of 200GPa. Unlike the fibres made via solvent route, tapes obtained in this publication show considerably high modulus but low tensile strength. To have insight in this discrepancy, tensile strengths against tensile modulus are plotted in logarithmic scale, for the samples synthesised at 30 minutes from heptane, toluene and the mixture (Figure 9). It should be noted that the same relationship holds also for all the other samples, but for simplicity only those at 30 minutes are shown in the graph, while the fitting data representing the constants $m$ and $n$ of equation 3 are reported in Table 3

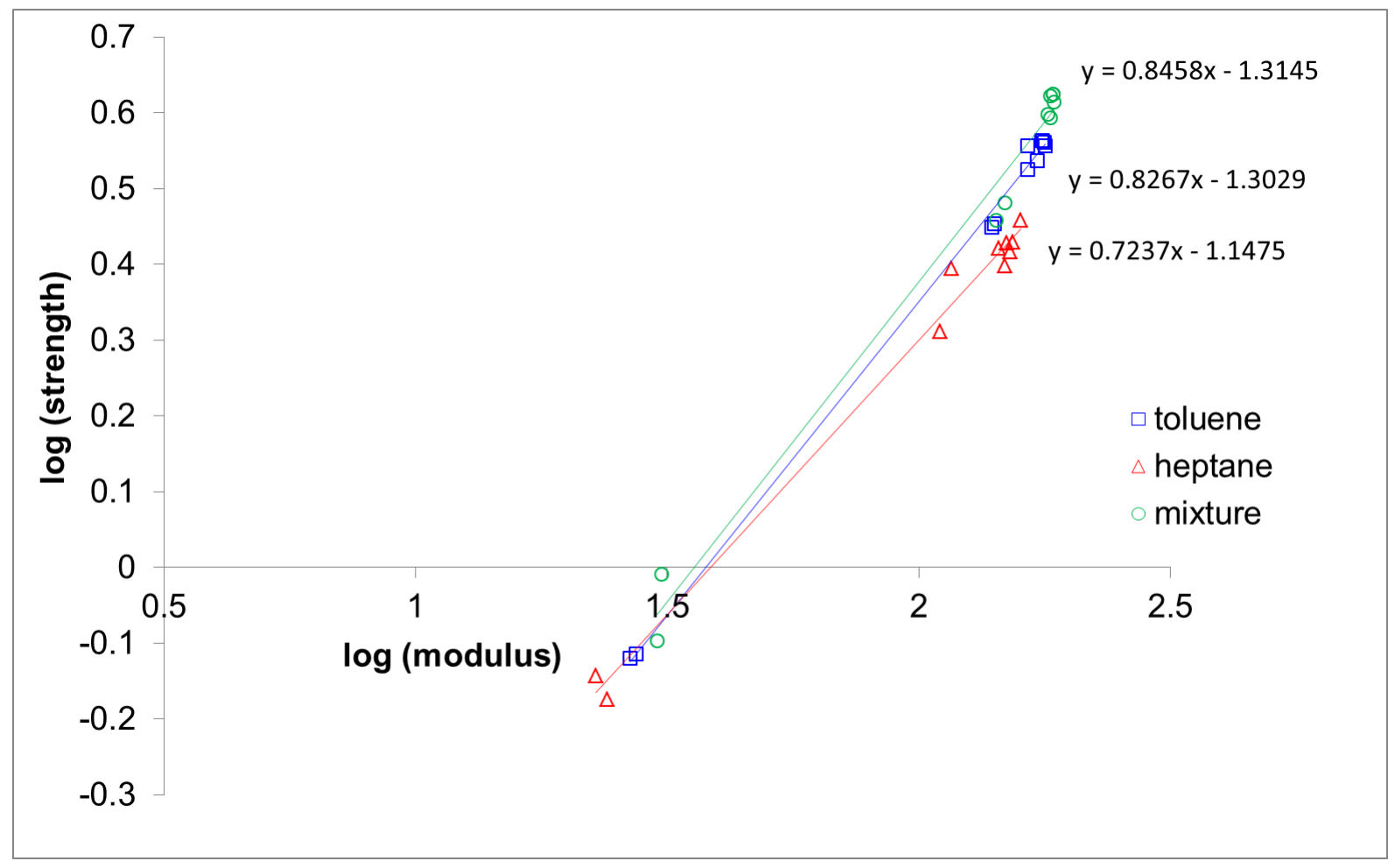

Figure 9. A linear relationship between log(tensile strength) and $\log$ (modulus) holds for samples having different molar masses and synthesised in different solvents. 
Independent of the polymerisation medium, all samples show the linear relationship between the tensile strength and tensile modulus confirming the relationship of equation 3 , where the constant $n$ falls within the anticipated range of 0.8 , once again surprisingly upholding the empirical relationship between tensile strength and tensile modulus in isotropic polymers proposed by van Krevelen in 1976 [18], which is also in accordance with our findings on tapes and the values reported for fibres by Smith and Lemstra [17].

A considerable discrepancy between the value of $m$ reported on fibres and that obtained in tapes is observed: Smith and Lemstra [17] reported values that increases from 0.08 to 0.15 when $M_{\mathrm{w}}$ increases from $8 \times 10^{5}$ to $4 \times 10^{6} \mathrm{~g} / \mathrm{mol}$, while in our case $\mathrm{m}$ ranges from 0.05 to 0.1 , with no apparent relationship to $M_{\mathrm{w}}$.

Table 3. Values of $m$ and $n$ as obtained from the linear fitting of the $\log$ (tensile strenght) versus $\log$ (modulus) for the various samples

\begin{tabular}{|l|l|l|l|l|}
\hline Sample & $\begin{array}{l}\text { Time } \\
(\mathbf{m} \text { in) }\end{array}$ & Solvent & $\mathbf{m}$ & $\mathbf{n}$ \\
\hline PE_2M_2.9 & 30 & $\mathrm{H}$ & 0.07 & 0.72 \\
\hline PE_4M_2.5 & 30 & $\mathrm{~T}$ & 0.05 & 0.83 \\
\hline PE_4M_2.8 & 30 & $\mathrm{M}$ & 0.05 & 0.84 \\
\hline PE_3M_3.0 & 60 & $\mathrm{H}$ & 0.10 & 0.68 \\
\hline PE_6M_3.2 & 60 & $\mathrm{~T}$ & 0.06 & 0.82 \\
\hline PE_7M_3.8 & 60 & $\mathrm{M}$ & 0.06 & 0.80 \\
\hline PE_4M_3.4 & 120 & $\mathrm{H}$ & 0.07 & 0.72 \\
\hline PE_7M_3.0 & 120 & $\mathrm{~T}$ & 0.06 & 0.79 \\
\hline PE_8M_3.4 & 120 & $\mathrm{M}$ & 0.05 & 0.83 \\
\hline
\end{tabular}


We should take into account that, as shown in one of our earlier publications, tapes are unique 'bundle' of fibres having a distinctive plane orientation that is inherited during the solid state processing. This unique crystal plane orientation is likely to provide a relatively high modulus compared to the fibres spun via solvent route, independently of the molar mass and molar mass distribution. However, the high tensile modulus inherited by the processing route cannot be upheld by the tensile strength, as it gets more vulnerable to the presence of voids and defects generated in the fibre bundle compared to a single strand of fiber.

\section{Conclusion}

In this work, we have used a homogenous single-site catalytic system to polymerize ethylene to linear UHMWPE having $M_{\mathrm{w}}$ up to 8 million $\mathrm{g} / \mathrm{mol}$ (MWD up to 3.4), with a reduced number of entanglements. The 'disentangled' nature of the polymer samples enable the solid-state processing of nascent polymer particles in coherent tapes that can be stretched to reach high modulus and strength. With a systematic set of samples having different $M_{\mathrm{w}}, M_{\mathrm{n}}$ and MWD we have been able to relate the molecular features to the mechanical properties of the obtained tapes. The results show that:

(a) The tensile strength of the UHMWPE tapes increases with increasing draw ratio, and beyond a critical draw ratio it enters a plateau region. The critical draw ratio increases with increasing molecular weight.

(b) The high tensile strength (in excess of $4 \mathrm{GPa}$ ) of the disentangled UHMWPE tapes can be attributed to high molar masses and narrow molecular weight distribution.

(c) The tensile modulus of the solid-state drawn tapes (greater than $170 \mathrm{GPa}$ for disentangled UHMWPE samples) is higher than the solution spun commercial fibres ( 130 GPa). The higher modulus in tapes can be attributed to the uniplanar-axial orientation that could be achieved through solid state processing [8]. 
(d) Trends correlating chain length and tensile strength can be identified; the available information suggests that tensile strength increases with chain length i.e. decreasing number of chain ends in the polymer having number average molar mass greater than a million $\mathrm{g} / \mathrm{mol}$.

\section{Methods}

Materials: All manipulations of air- and moisture-sensitive compounds were performed under nitrogen or argon atmosphere using standard high-vacuum Schlenk techniques or in a glovebox. Toluene (99.8\%, anhydrous) and Methylaluminoxane (MAO, 10\%wt solution in toluene) were purchased from Aldrich, ethylene (grade 3.0) was purchased from BOC and FI precatalyst bis[N-(3-tert-butylsalicylidene)pentafluoroanilinato]titanium (IV) was purchased from MCat. All reagents were used as received.

Synthesis of disentangled UHMWPE: A wall-mounted, 10L jacketed Pyrex reactor was equipped with a double plane propeller blade mechanical stirrer, a temperature probe, a gas inlet/outlet and a rubber septum for catalyst injection. The reactor was kept overnight at $125^{\circ} \mathrm{C}$, then the temperature was brought to room temperature while the vessel was purged with three cycles of vacuum/nitrogen. The required amount of solvent (toluene, heptane or a mixture of them) was transferred under a nitrogen gas stream into the vessel and the temperature was set at the desired value $\left(10^{\circ} \mathrm{C}\right)$ by mean of a thermostat. When the desired temperature was reached, the stream of gas was switched from nitrogen to ethylene. Ethylene uptake was controlled by mean of a Buchi press flow gas controller bpc 6002 . When the solution was saturated with ethylene at the desired partial pressure (1 bar ethylene), MAO solution was added, followed by a solution of the catalyst in toluene + MAO to start the polymerization. The reaction was carried on for the required time under vigorous stirring and constant feed of ethylene, then quenched by addition of methanol. The polymer was filtered out, washed with additional methanol and oven-dried under vacuum at $40^{\circ} \mathrm{C}$ for one night. 
Polymer processing of nascent disentangled and entangled powders: A general procedure for the preparation of tapes is as follows: $25 \mathrm{~g}$ of polymer powder is poured into a mold with a cavity of $620 \mathrm{~mm}$ in length and $30 \mathrm{~mm}$ in width and compression molded at 130 bar for $10 \mathrm{~min}$ to form a sheet. The sheet is preheated for at least $1 \mathrm{~min}$ and rolled with a Collin Calander (diameter rolls: $250 \mathrm{~mm}$, slit distance $0.15 \mathrm{~mm}$, inlet speed $0.5 \mathrm{~m} / \mathrm{min}$ ). The tape is immediately stretched on a roll (speed $2.5 \mathrm{~m} / \mathrm{min}$ ). The rolled and stretched tape is further stretched in two steps on a 50 $\mathrm{cm}$ long oil heated hotplate. The tape comes in contact with the hotplate after $20 \mathrm{~cm}$ from the entrance of the hotplate. The draw ratio is obtained by dividing specific weight of the sheet prior to deformation by the specific weight of the tape after stretching. Typical processing temperatures are given in reference 8.

Tensile testing: Tensile properties are measured using an Instron 5566 tensile tester at room temperature $\left(25^{\circ} \mathrm{C}\right)$. To avoid any slippage the side action grip clamps with flat jaw faces are used. The nominal gauge length of the specimen is $100 \mathrm{~mm}$ and the test is performed at a constant rate of extension (crosshead travel rate) $50 \mathrm{~mm} / \mathrm{min}$. The breaking tenacity (or tensile strength) and modulus (segment between $0.3-0.4 \mathrm{~N} /$ tex) are determined from the force against displacement between the jaws.

Rheology: To perform the rheological studies a procedure similar to the one described in ref. 9 and 12 is applied. The nascent powder is compressed into a plate of $50 \mathrm{~mm}$ diameter and thickness $0.6-0.7 \mathrm{~mm}$ at a fixed temperature of $125^{\circ} \mathrm{C}$, under a maximum force of 20 tons for an average time of 20 minutes. From the compressed plate, using a punching device, $12 \mathrm{~mm}$ diameter discs are cut for rheological studies. Rheological experiments are performed using a $12 \mathrm{~mm}$ parallel plate strain controlled rheometer TA instruments, ARES G2 or ARES LS2. The disc is placed between parallel plates at an initial temperature of $110^{\circ} \mathrm{C}$. To prevent oxidation, the temperature is controlled by a convection oven under a nitrogen environment. After thermal stabilization at $110^{\circ} \mathrm{C}$, temperature is increased to $130^{\circ} \mathrm{C}$ at $30^{\circ} \mathrm{C} / \mathrm{min}$. After the stabilization, the sample is heated further to $160^{\circ} \mathrm{C}$ at $10^{\circ} \mathrm{C} / \mathrm{min}$ while maintaining the compression force of 
$4 \mathrm{~N}$. Subsequently, an oscillatory amplitude sweep test is carried out to determine the range of oscillatory strains in the linear viscoelastic region. The test is performed at a fixed frequency of $10 \mathrm{rad} / \mathrm{s}$. To follow changes in the modulus of the polymer melt, during the transformation from non-equilibrium to equilibrium state, an oscillatory time sweep is performed; the test is executed at a fixed frequency of $10 \mathrm{rad} / \mathrm{s}$ and a strain between $0.1-0.5 \%$, well within the linear viscoelastic regime. To determine the molecular weight of the polymer, after reaching the storage plateau modulus, a small amplitude oscillatory frequency sweep test at a fixed strain between 0.3-0.5\% is performed. Using the commercial TA Orchestrator software available, based on the method developed by Mead $^{19}$, molecular weight and molecular weight distribution are determined for linear polyethylene. Frequency sweep tests are performed in a range of 100 to $1 \times 10^{-3} \mathrm{rad} / \mathrm{s}$, at a constant strain $(0.3-0.5 \%)$ within the linear viscoelastic region of the polymer. Using the TA Orchestrator software available on the rheometer, molecular weight and molecular weight distribution were estimated.

\section{Acknowledgements}

The authors wish to thanks Ir. Satyan Choudhary, Teijin Aramid, The Netherlands for rheological experiments. This work was supported by EPSRC (grant EP/K034405/1).

\section{Competing financial interests}

The author(s) declare no competing financial interests. 


\section{References}

1] Capaccio, G., Ward, I.M. Effect of molecular weight on the morphology and drawing behaviour of melt crystallized linear polyethylene Polymer 2005, 16, 239-243.

2] Flory, P.J. On the morphology of the crystalline state in polymers J. Am. Chem. Soc. 1962, 84, 2857-2867.

3] Yao, Y., Jiang, S., Rastogi, S. 13C Solid State NMR Characterization of Structure and Orientation Development in the Narrow and Broad Molar Mass Disentangled UHMWPE Macromolecules 2014, 47, 1371-1382.

4] Smith, P., Lemstra, P., Booij, H.C. Ultradrawing of high-molecular-weight polyethylene cast from solution. II. Influence of initial polymer concentration J. Pol. Sci.: Pol. Phys. 1981, 19, 877888.

5] Ward, I.M. Processing. Structure and Properties of Oriented Polymers Makromol. Chem., Macromol. Symp. 1988, 22, 59-82.

6] Smith, P., Chanzy, H.D., Rotzinger, B.P. Drawing of virgin ultrahigh molecular weight polyethylene: an alternative route to high strength/high modulus materials J. Mater. Sci. 1987, 22, 523-531.

7] Rastogi, S., Lippits, D., Peters, G.R., Graf, R.W.M., Yao, Y., Spiess, H. Heterogeneity in polymer melts from melting of polymer crystals Nat. Mater. 2005, 4, 635-641.

8] Rastogi, S., Yao, Y., Ronca, S., Bos, J., van der Eem, J. Unprecedented high-modulus highstrength tapes and films of ultrahigh molecular weight polyethylene via solvent-free route Macromolecules 2011, 44, 5558-5568.

9] Pandey, A., Champouret, Y., Rastogi, S. Heterogeneity in the distribution of entanglement density during polymerization in disentangled ultrahigh molecular weight polyethylene Macromolecules 2011, 44, 4952-4960.

10 ] Smith, P., Lemstra, P.J. Ultra-high-strength polyethylene filaments by solution spinning/drawing J. Mater. Sci., 1980, 15, 505-514.

11] Nishii, K., Matsumae, T., Dare, E.O., Shiono, T., Ikeda, T. Effect of Solvents on Living Polymerization of Propylene with [t-BuNSiMe2Flu] TiMe2-MMAO Catalyst System Macromol. Chem. Phys. 2004, 205, 363-369. 
12] Talebi, S., Duchateau, R., Rastogi, S., Kaschta, J., Peters, G.W.M., Lemstra, P.J. Molar mass and molecular weight distribution determination of UHMWPE synthesized using a living homogeneous catalyst Macromolecules 2010, 43, 2780-2788.

13] Kremer, K. Statics and dynamics of polymeric melts: a numerical analysis Macromolecules 1983, 16, 1632-1638.

14] Termonia, Y., Meakin, P., Smith, P. Theoretical study of the influence of the molecular weight on the maximum tensile strength of polymer fibers Macromolecules 1985, 18, 22462252.

15] Termonia, Y., Meakin, P., Smith, P. Theoretical study of the influence of strain rate and temperature on the maximum strength of perfectly ordered and oriented polyethylene Macromolecules 1986, 19, 154-159.

16] Savitsky, A.V., Gorshkova, I.A., Frolova, I.L., Shmikk, G.N., loffe, A.E. The model of polymer orientation strengthening and production of ultra-high strength fibers Pol. Bull. 1984, 12, 195202.

17] Smith, P., Lemstra, P.J. Tensile strength of highly oriented polyethylene J. Pol. Sci.: Pol. Phys. 1981, 19, 1007-1009.

18] van Krevelen, D.W. Properties of Polymers, 2nd ed., Elsevier, Amsterdam, 300 (1976).

19] Mead, D. W. J. Rheo. 1994, 38, 1797-1827 


\section{Figure and table legends}

Table 1. Molecular characteristics of the UHMWPE samples synthesised in toluene at various times (tp).

Figure 1. Modulus build-up as a function of time for samples synthesized at different reaction times.

Figure 2. Frequency sweep data showing the progressive shift of the cross-over point to lower frequencies with increasing $M_{\mathrm{w}}$.

Figure 3. Molar mass distribution curves for the polymer of Table 1, as obtained from frequency sweep data.

Figure 4 a) tube renewal time (obtained from the frequency cross-over point) as a function of $M_{\mathrm{w}}{ }^{3}$ and b) modulus build up time (time by which the polymer modulus has reached the plateau region) as a function of $M_{\mathrm{w}}$.

Figure 5. Influence of draw ratio on tensile strength of disentangled UHMWPE tapes. (Tex is defined as the mass in grams $\backslash 1000$ meters). Lines are drawn for visual guidance only.

Figure 6. Influence of draw ratio on tensile modulus (N/Tex) for different polymerization time (Tex is defined as the mass in grams per 1000 meters). Lines are drawn for visual guidance only.

Table 2. Molecular characteristics of the UHMWPE samples synthesised in heptane $(H)$ and heptane/toluene (25/75 volume ratio) mixture (Mix).

Figure 7. Overview of molar masses and molecular weight distribution for all the samples considered in this study.

Figure 8. Tensile strength (top) and modulus (bottom) as a function of draw ratio for samples obtained from $(\mathrm{H})$ heptane, $(\mathrm{T})$ toluene and $(\mathrm{M})$ mixture of both solvents. For clarity, only the samples from 30 and 60 minutes reaction are shown. 
Figure 9. A linear relationship between log(tensile strength) and log(modulus) holds for samples having different molar masses and synthesised in different solvents.

Table 3. Values of $m$ and $n$ as obtained from the linear fitting of the $\log$ (tensile strenght) versus $\log$ (modulus) for the various samples. 